\title{
Pigeon Metheuristic Optimized Generative Adversarial Networks and ARKFCM Algorithms for retinal Vessel Segmentation and Classification
}

\author{
R. Kiran Kumar, K. Arunabhaskar, Ch. Mani Mala
}

\begin{abstract}
Automatic evaluation of retinal vessels acts a significant part in diagnosis of several ocular and systemic diseases. Eye diseases must be diagnosed early to avoid severe infection and vision loss. The method of segmentation and classification of the retinal blood vessel identification is most difficult tasks in computerized fundus imaging now a days. To solve this problem in this paper, to locate retinal vessel in the retinal vessel, Adaptive Regularized Kernel Based Fuzzy Clustering Means (ARKFCM) algorithm-based segmentation is used. For retinal vessel prediction purpose in this paper a PIGEON optimization-based learning rate modified Generative Adversarial Networks (GAN) algorithm is introduced. Additionally, to improve the proposed classification performance input image is transformed with the aid of Discrete Wavelet Transform (DWT). The DWT applied Low Low (LL) image and segmented images are cascaded. The cascade images are used for training and testing. The proposed system has validated with the help of DRIVE and STARE publically available datasets. They are studied by applying a Convolutional Neural Network, an instantly trained neural network for predicting retinal vessel. In the end, the system is checked for system efficiency using the results of modeling based on MATLAB. The scheme guarantees an accuracy of $92.77 \%$ on DRIVE dataset and $98.85 \%$ on STARE dataset with a minimum average classification error of $2.57 \%$. Further, we recommended to physician for implement the real time clinical application; this scheme is highly beneficial for doctors for identifying retinal blood vessels.
\end{abstract}

Keywords: Discrete Wavelet Transform (DWT), Adaptive Regularized Kernel Based Fuzzy Clustering Means (ARKFCM), Diabetic Retinopathy (DR), and modified Generative Adversarial Networks (GAN) algorithm.

Manuscript received on November 10, 2021.

Revised Manuscript received on November 15, 2021.

Manuscript published on November 30, 2021.

* Correspondence Author Krishna University, Machilipatnam (AP), India. Email: kirankreddy@gmail.com

K. Arunabhaskar*, Department of Information Technology, Aditya Engineering College, Surampalem (AP), India. Email: letter2arunbhaskar@arec.edu.in

Dr. CH. Mani Mala, MBBS, DO, FCO (LVPEI), Senior Consultant \& Phaco Surgeon, Vasan Eye Care, Kakinada (AP), India. Email: dr.manimalaa@gmail.com

(c) The Authors. Published by Blue Eyes Intelligence Engineering and Sciences Publication (BEIESP). This is an open access article under the CC BY-NC-ND license (http://creativecommons.org/licenses/by-nc-nd/4.0/)
Dr. R. Kiran Kumar, Department of Computer Science \& Engineering,

\section{INTRODUCTION}

$D_{\text {iabetic retinopathy is a diabetes difficulty that affects }}$ eyes. It caused by the retinal blood vessels that happens as a result of diabetes. There are retinal surgeries that can relieve symptoms, but controlling diabetes and treating early symptoms are the most effective ways to prevent DR. The retinal arrangement of DR eyes can be seen using the fundus imaging procedure. These images are basically captured by a camera that focuses through the eye. The membranes of a healthy eye are just blood vessels that contain blood and nutrients from the eye. In diabetes, these blood vessels are basically fragile and broken due to high blood pressure. The progression of DR due to excessive pressure can be caused by the proliferation of small foreign blood vessels from the surface of the retina. This increase in blood vessels relative to the normal retina can be used as a biomarker to classify diverse phases of $\mathrm{DR}$, such as proliferative diabetic retinopathy (PDR) and Non-proliferative diabetic retinopathy (NPDR). [1]. Over the past decade, many investigators have developed efficient vascular segmentation schemes and classified retinal images based on the severity and type of disease. [2-5]. Nayak et al., [6] Projected an automated method to classify retinopathy based on artificial neural networks for early detection of DR. This method uses a morphological operator to separate blood vessels and exudates. In the second method, Fuzzy C Means values (FCM) and genetic algorithms are known as exudates with an accuracy of 96\%. [7]. Akram et al. [8] obtainable a multilayered thresholding practice to blood vessels segment in retinopathy imageries. Wavelet [9], ridgelets [10] and curvelet [11] Transformers with fundus images are also used to analyze retinal structures. The obscure logic of detecting hard exudates in DR images achieved a sensitivity of $99.9 \%$ [12]. In 2012, Nguyen et al. [13] established a retinal vascular feature analysis using a multi-scale line detector. Roychowdhury et al. [14] established a DREAM (DR analysis using machine learning) project by joining the Gaussian mixer ideal with the k-nn procedure and classified. Additional, Zhao et al. [15] used L2 Lebesgue integral method to estimate an infinite perimeter regularization.

Published By:

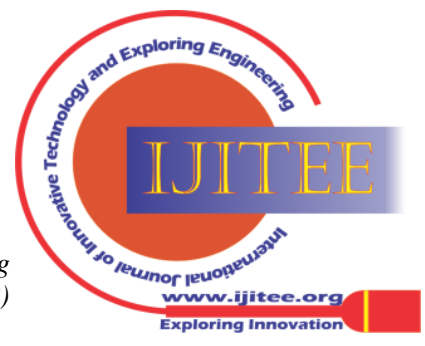




\section{Pigeon Metheuristic Optimized Generative Adversarial Networks and ARKFCM Algorithms for retinal vessel Segmentation and Classification}

Global thresholding system is implemented by Mapayi et al. [16] for preprocessing of retinal images. The Morphological Component Analysis (MCA) of Ship Detection by using the Stars and Drive Datasets was obtained accuracy of $96.23 \%$ and $95.23 \%$ respectively [17]. Premature retinopathy in blood vessels can be studied from the level of the turtle above the retina. Masoud et al. [18] Introduced curvature-based algorithms to classify ships by turtle level. Roychowdhury et al. [19] boundary of the optic nerve head was assessed using the Gaussian mixing model. Liskowski and Krawiec [20] used a deep nerve network method that trained on huge trials of units, stars, and chest datasets. However, this method requires a significant amount of samples (up to 400,000). In a latest work, Seoud et al. [21] development of a telemedicine system using readiness for additional screening of retinopathy images on a computer. A straightforward method of establishing a classification for reference to DR was then proposed. [22]. Wound removal reduced the effort required to identify injuries, but a large amount of data is needed to train classification. All existing methods, such as neural networks, and other computer intelligence, have come a long way in finding efficient and accurate DR as quickly as possible. Before we discussed some previous existing techniques in retinal blood vessels classification models, here we find the major drawbacks of the existing methods as low learning rate,However, these methods are very complex and require a lot of training to classify. For the purposes of previous work in this article, we have introduced a new and simple method to overcome that the problem by using the proposed classification techniques to provide better learning rate and improved performance than existing techniques. robotically diagnoses diabetic retinopathy is defined. A total of 1616 people were photographed. Retinal image were assessed using hybrid enhanced deep learning tools and also assessed using reference standards. The hybrid improved deep learning instrument achieved $99 \%$ and $97.8 \%$ specificity compared to the vision-threatening DR standards and $79.4 \%$ sensitivity compared to more than mild [mtm] DR device and 93 specificity reached $8 \%$ of the reference standards. It showed that hybrid deep learning improved the method and ensured high detection accuracy of vtDR and mtmDR. [13]. T. Jebaseeli, et al., [24] have introduced the novel technique to exactly segment the pathological retinal images. In this techniqueholds the different technique as CLAHE is for pre-processing and extraction process of the retinal blood vessels via Deep Learning Based Support Vector Machine (DLBSVM)..The anticipated devices were accomplished to piece all minute BV pixels deprived of rebelliousness. The unvarying supply of grey morals boosts the fundus carbon copy and kinds the covered structures further evident. Cycle Beat Attached Neural Network model regenerates the wrecked inter-sections vessel, overlying borderline vessels, and attach with its consistent receptacles tripe. The optional tactic was considered of the normal public fundus image appraise the concert in the file. The investigational results stated the technique that technique achieved nearly $99.45 \%$ for both specificity and accuracy of $74.45 \%$ for sensitivity to the accurate segmentation. This grind can be protracted by pull out the vessel topographies
Verbraak, F. D. et al. [23] defined the device that

and to amount the tortuosity built on its landscapes cataloguing. Pires, R. et al. [25] information extracted from retinal images. In this context, neural networks were implemented. This study extracted features that examined the patient and tried to identify deficiencies. The proposed technique preserved the receiver operating specific area of curve (ROC) value of $2.2 \%$. Applies the indicated technique to the Messidor-2 dataset. Alike outcomes were gained using two to five times the cross-validation protocol when practical to the Messidor-2 - ADCISand DR2 datasets. When rating recommendations, this technique gave $99.0 \%$ the highest results when using the Convoluted Neural Network (CNN).

S. Dutta, et al., [26] offered a mechanical knowledge perfect to recognize the key backgrounds of DR. The proposed techniques with three types such as back propagation NN, Deep Neural Network (DNN) and CNN deep learning method to classify the DR images exactly. The Deep Learning facsimiles were adept of enumerating the structures as BV, fluid drip, exudates, haemorrhages and micro aneurysms into changed courses. Exemplary resolve estimate the masses which gave ruthlessness near of the patient's eye. The accurate ruling for thresholds of separate part class was the main experiment of this learning, where weighted FCM system was used to recognize the target class threshold. The rigorousness of accurate class of DR images were well-known by using this model. For imminent work model can Inter City with GPU classification, with supplementary quantity of accomplished data for getting difficult correctness consequence. To stun the above concerns, the wished-for method implemented the hybrid algorithm used for both integration and grouping for retinal BV. T. Jebaseeli, et al., [27] wished-for an organisation towards the excellence of the separation outcome done in the ophthalmologic Diabetic Retinopathy images. The planned technique charity CLAHE is for pre-processing and classification and extraction of the retinal blood vessels through DLBSVM. The anticipated devices were accomplished to piece all minute BV pixels deprived of rebelliousness. The unvarying supply of grey morals boosts the fundus carbon copy and kinds the covered structures further evident. Cycle Beat Attached Neural Network model regenerates the wrecked inter-sections vessel, overlying borderline vessels, and attach with its consistent receptacles tripe. The optional tactic was considered of the normal public fundus image appraise the concert in the file. The investigational results stated the technique that technique achieved nearly $99.45 \%$ for both specificity and accuracy of $74.45 \%$ for sensitivity to the accurate segmentation.

\section{MATIRALS AND METHODS}

In this study, two datasets as DRIVE and STARE dataset are using. Both dataset descriptions are briefly explained in following section 3.1. The processing of the proposed method is followed as in training section, dataset images are initially preprocessed by removing noises in the original images.

Published By:

Blue Eyes Intelligence Engineering and Sciences Publication (BEIESP)

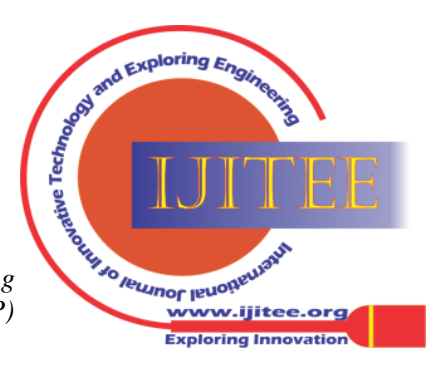


Then the Preprocessed images are given to segmentation process by using Adaptive regularized kernel based fuzzy clustering means techniques. After that the segmented images is given to the proposed classifier. On other hand, testing section same as the training section. After segmentation process, we used discrete wavelet transform technique to extract the feature from dataset images. This dataset images is given to the proposed classifier as a PIGEON optimization based learning rate modified Generative Adversarial Networks classification algorithm. This section accurately classifies to predicting retinal vessel as normal or infected.

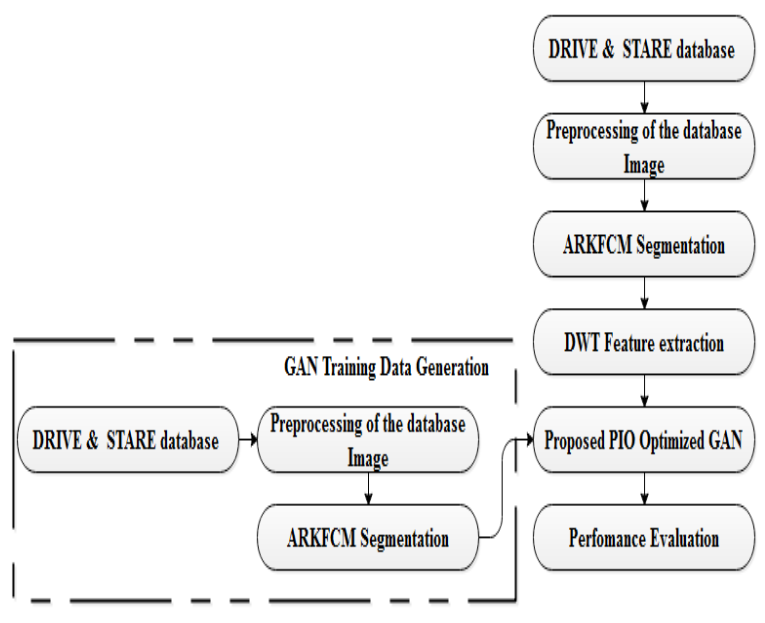

Fig 1: Proposed flow diagram.

The proposed scheme has been analyzed with the assistance of two kind of databases such as DRIVE and STARE the description of the databases are detailed in the below section.

\section{i. DRIVE database}

The drive dataset is most commonly used for partition records for retina sections. This is a freely available data set with 40 color images from fundus photographers, of which 33 show no signs of DR and 7 show signs of mild DR. The images were taken with a Canon CR53 CCD camera with 8 bit $768 * 584$ pixels (per color plane) [1]. The data set is divided into two sets, one is a test set and the other is a training set with twenty images each.

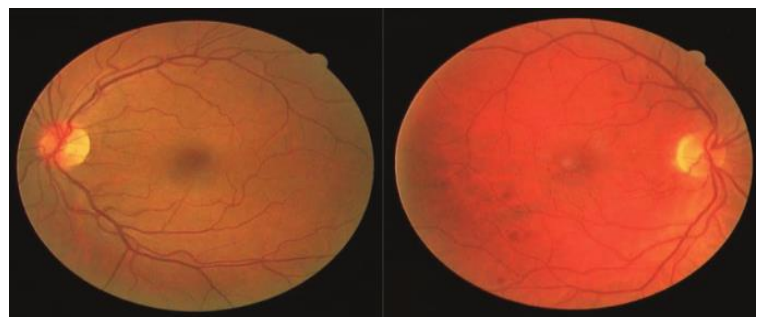

Fig 2: Sample image from DRIVE dataset.

\section{ii. STARE database}

1) It comprises 20 color retinal images. The images of 10 are healthy images, then another 10 images are pathological images of the retina. Images are taken by Funds Camera Topcon-50 at 35 FOV. Two observers manually segmented all images in the STARE database and gave the boat pixel percentages at 10.4 and 14.9 , correspondingly.

\section{A.Dataset}
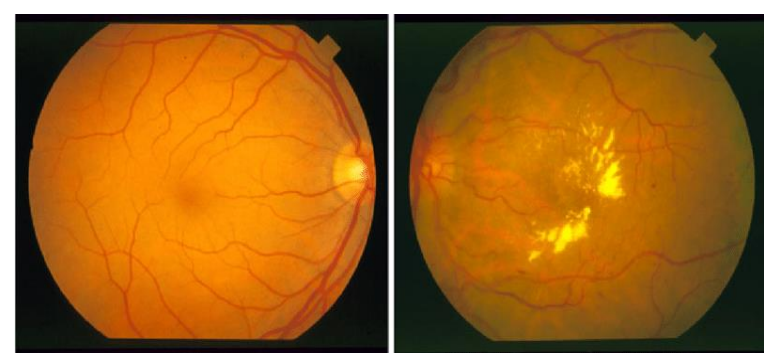

Fig 3: Sample images from STARE database

\section{B. Preprocessing of the Input Image}

To eliminate the noise and need fine images for this development so the firstly period of splitting up procedure is pre-processing. To find the good results, the pre-processing step plays a main role, where gray scale images are gained by transforming the RGB image. Therefore, it makes these images are furtherseemly for the integration process. The countless footsteps done the RGB spitting image embraces in preprocessing stage:- Green Constituent are removed from RGB Image: - The effort image contains of three stations as that rendering of each RGB image, but the green frequency holds the extreme data and distinction concerning to green constituent image. Input image sideways with that diverse station is presented in Figure 4. It changes into gray -the image of gauge and sifting: - For declining the difficulty of the image, the preprocessed image is transformed which also improved its ability for segmentation. As there is uneven enlightenment and have noise in dual input, so certain filtering method is needed for noise removal. The image displayed in Figure 4(a) is after adaptation into Gaussian riddling and gray scale. In contrast Enhancement: -Range of image grows with the active range, CLAHE method is used and further contrast the image in the beneath attention. After the way of pre-processing, the input image is right for it added dissection route.

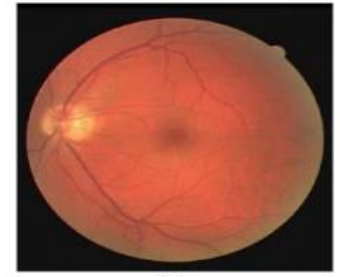

(a)

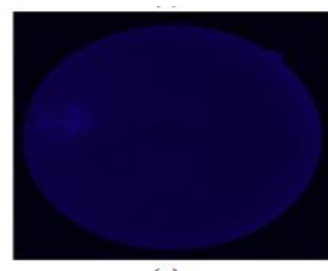

(c)

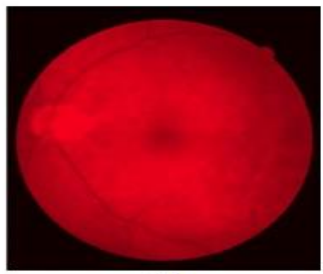

(b)

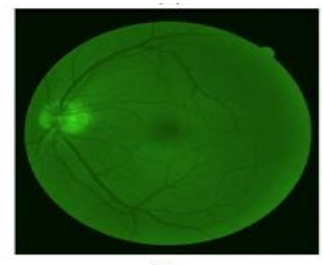

(d)
Fig 4: A) Original RGB, B) Red station, C) Blue Station D) Green Station.

\section{Segmentation}

Retinal images usually contain normal and abnormal segments. Normal and unusual identification are crucial for accurately extracting features.

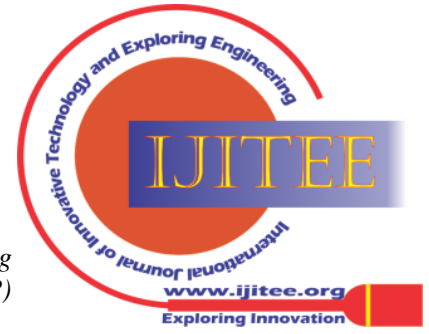




\section{Pigeon Metheuristic Optimized Generative Adversarial Networks and ARKFCM Algorithms for retinal vessel Segmentation and Classification}

Retina blood vessel can be identified using segmentation techniques. The proposed kernel based FCM framework considers the proposed system called Adaptive regularized kernel based fuzzy clustering means (ARKFCM). Which is applied to remove nuclei and non-nuclei images of the histopathological ROI image. In ARKFCM, the results occurred in two sets of images, both images are examined to generate grouping cells in this system, first, we calculate the flexible parameter Lex $\varphi a_{-} a$ for each pixel to control the contextual information using in equation (1). Objective function is defined as below equation.

$J_{\text {ARKFCM }}=2\left[\sum_{a=1}^{i} \sum_{b=1}^{c} u_{a b}^{m}\left(1-K\left(m_{a}, v_{b}\right)\right)+\sum_{a=1}^{i} \sum_{b=1}^{c} \varphi_{a} u_{a b}^{m}\left(1-K\left(\bar{m}_{a}, v_{b}\right)\right)\right]$

Where, $a$ indicate the row of the image and $b$ indicate the column of the image. The minimization of $7_{\text {ARKFCM }}\left(m_{v} v\right)$ can be computed through another optimization process using

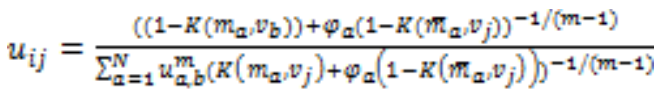

$$
\begin{aligned}
& v_{a}=\frac{\sum_{a=1}^{N} w_{a b}^{m}\left(K\left(m_{a}, v_{b}\right) m_{a}+\varphi_{a} K\left(m_{a}, v_{j}\right) m_{a}\right)}{\sum_{a=1}^{N} w_{a b}^{m}\left(K\left(m_{a}, v_{b}\right) m_{a}+\varphi_{a} K\left(m_{a} v_{j}\right)\right)}
\end{aligned}
$$

When $\bar{x}$ is swapped with the average filter of the input the grayscale image, the procedure is indicated as ARKFCM. When $\bar{x}_{i}$ is swapped with the weight image $\bar{\varepsilon}_{i}$ defined. The algorithm is denoted as ARKFCM $_{\omega}$.

The main step for the ARKFCM is mentioned below:

\section{- ARKFCM algorithm :}

(1) Set threshold value $\varepsilon=0.001, m=2$, loop counter $t=0, v$ and $u^{(0)}$

(2) Compute the regularization parameter $\varphi_{i^{x}}$

(3) Compute $\bar{x}_{i}$ for ARKFCM

(4) Compute cluster centers $v_{j}^{(t)}$ using $u^{(t)}$ as in (1).

Compute the membership function $u^{(t+1)}$ within (1)

If max $\| u^{(t+1)}-u^{t} \mid<\varepsilon$ or $t>100$ then stop; else, update $t=t+1$ and move to phase (4).

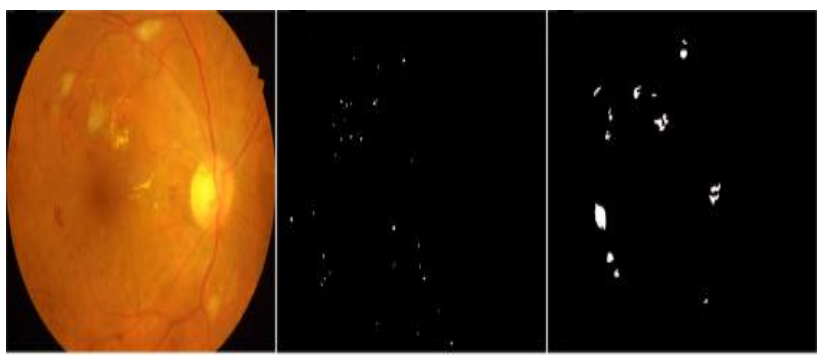

(a) Input image.

(b) Cluster 1 sample. (c) Cluster 2 sample.

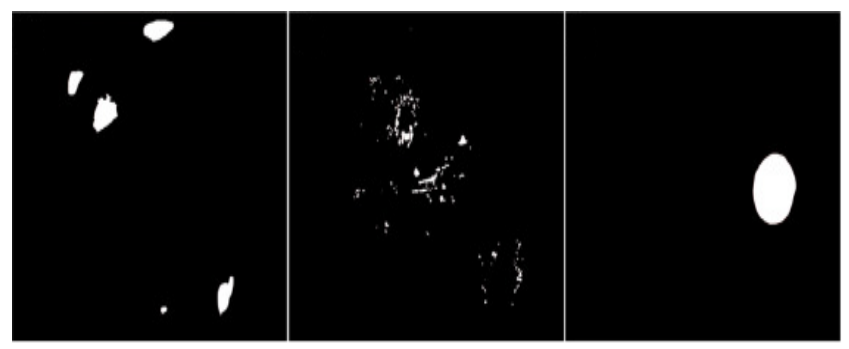

(d) Cluster 3 sample. (e) Cluster 4 sample. (f) Cluster 5 sample.

Fig. 5: ARKFCM based Segmentation.

\section{Wavelet-Based Features}

In short, the Discrete wavelet Transform (DWT) functions are linear transformers that are applied to a data vector, which are converted to the same quantitative values. This means that the data is divided with different frequency resolutions. Then, the DWT value is calculated by sequential flow using an undersampling factor of two, i.e. high pass filter $(\mathrm{H})$ and low pass filter $(\mathrm{L})$. For this study, the haar wavelet is considered for the data set. This data set is divided into four sub-bands (HH, HL, LH and LL) on each scale. The LL sub-band is used to calculate DWT properties.

\section{E. Generative Adversarial Networks}

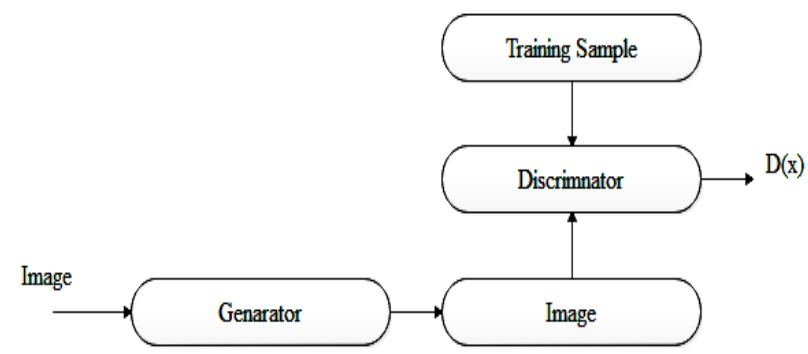

Fig. 6: Architecture of GAN

GAN is based on a zero-sum game. In short, one wins and the other loses. The zero sum game is also called Minimax. Your participants want to exploit their actions and minimize your actions. In below summary of the statistics of how we train the generator and discriminator using the correct diagram. The GAN architecture is expressed in fig.6.

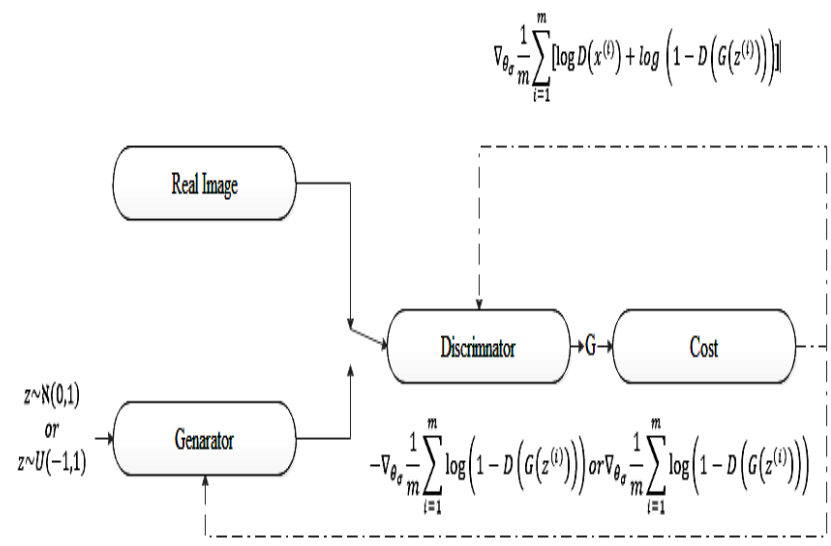

Fig.7. GAN Training flow diagram.

In game theory, the GAN model is converted when the discriminator and the Nash generator touch equilibrium. Below is the optimum point for the minimax equation.

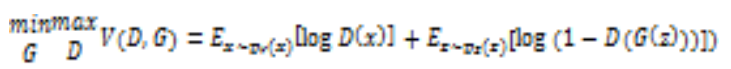

Since both sides want to demoralize each other, Nash equilibrium occurs when the player does not change his actions no matter what the opponent is allowed to do. Consider two players, A and B, controlling the values of $\boldsymbol{x}$ and $\boldsymbol{y}$, correspondingly. Player A wants to exploitxy and $\boldsymbol{B}$ wants to reduce it.

$\min _{B} A \mathrm{vax}(\mathrm{D}, \mathrm{G})=\mathrm{xy}$

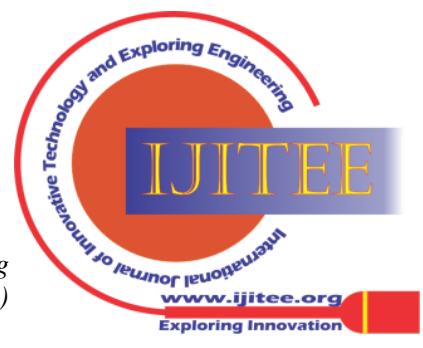


The Nash equilibrium is $\boldsymbol{x}=\boldsymbol{y}=\mathbf{0}$. This is the only state in which your opponent's actions are irrelevant. This is the only condition that an opponent's action does not changthe outcome of the game.

Let's see if we can easily find the equilibrium using the gradient. We apprise the $\mathrm{x}$ and $\mathrm{y}$ parameters according to the gradient of the $\mathrm{V}$ value function.

$\Delta x=a \frac{\Delta(x y)}{\Delta(x)}$

$\Delta y=a \frac{\Delta(x y)}{\Delta(y)}$

Where $\boldsymbol{\alpha}$ is the learning rate. When we design $\mathrm{x}, \mathrm{y}$ and xy against the training iterations, we understand our key does not converge. To improve the learning rate in this work Pigeon bio-inspired (PIO) algorithms is used the working of the PIO is defined in the below section.

\section{F. Pigeon bio-inspired algorithm}

PIO algorithms have recently been exposed to be effective in solving various optimization issues, including aerial robot trajectory planning, three-dimensional trajectory planning, an automatic landing system, and a PID development controller. In this article, we adopt the Learning rate selection on GAN network based on the fresh binary version of PIO. This unit offers two versions of the PIO. The first version or algorithm uses a sigmoid function to sample the speed of the doves, the second forms offers an updated improved binary version of the basic PIO, which uses cosine similarity to determine the speed of the doves. Both versions use an equal fitness function, another each form has methods that represent a pigeon or a solution.

\section{i. Fitness Function}

It is the terms of a process for evaluating the sufficiency of solutions. The fitness function evaluates the solution, which is a subset of the functions selected according to the true positive speed (TPS), the false positive speed (FPS), and the sum of functions. The sum of functions is involved in the adaptation function, so, if there are few functions that does not disturb the TPS or FPS, we want to avoid it. Eq. 7 represents the formula used to calculate the taste of a dove or solution. Here is the sum of objects chooses, the total number of objects in FP and FN is $\boldsymbol{w} \mathbf{1}+\boldsymbol{w} \mathbf{2}+\boldsymbol{w} \mathbf{3}=\mathbf{1}$. The weight is set as follows: $\boldsymbol{w 1}=0.1, w 2=w 3=0.45$, because TPS and FPS are equal.

$F F=w_{1} * \frac{S F}{N F}+w_{2} * F P S+w_{3} * \frac{1}{T P S}$

\section{ii. SIGMOID PIO for FS}

Sigmoid PIO for Fs is used to define a solution or a pigeon vector of length equal to the sum of training data. Since the basic PIO procedure continuously processes the dove's position, the specific PIO solution for the learning rate is defined as a vector whose values of velocity and position vectors are fixed randomly among initial $[0,1]$. The traditional method is used to measure the rapidity of every pigeon according to Equ. (8), and then the sigmoid function is used to translate the velocity into a binary version according to Equation 8. For the binary files of the cluster intelligence algorithm, the location of each dove is updated based on the value of the sigmoid function and the probability of a uniform random numeral between $[0,1]$ according to Equation 9 . The algorithm will act as an old PIO, except for updating the position of the ground operator. Additionally, the sigmoid function will be used to transferal the speed, and then the locations will be informed accordingly.

$$
S\left(V_{i}(t)\right)=\frac{1}{1+e^{\frac{-\pi i}{2}}}
$$

$$
X(t)_{\text {(ip })}[i]=\left\{\begin{array}{cc}
\mathbf{1}_{s} & \text { if }\left(S\left(V_{i}(t)\right)>r\right) \\
0, & \text { otherwish }
\end{array}\right.
$$

\section{RESULTS AND DISCUSSION}

Experimental setup that, the proposed method is computer with in 8GB RAM was implemented, In MATLAB R 2018b by using Intel i7 with $2.7 \mathrm{GHz}$. The validation of proposed system was verified using the parameter metrics such as accuracy, sensitivity, specificity and F-measure. The below section explains the dataset used for validating the proposed system, parameter evaluation, qualitative analysis of proposed method against existing techniques.

\section{A. Evaluation Parameters}

The proposed system is validated against surviving techniques by using several parameters, which are discussed in this section. The estimation metrics are there used to check the property of the proposed system to explain notional and hands-on advances schemes. It comprises, that set of measures that follow a common original attitude of evaluation. The estimate metrics are sensitivity, specificity, f-measure, Accuracy. The equations used for spread over this metrics measures are given below,

$$
\begin{aligned}
& \text { Sensitivity }=\frac{T P}{T P+F N} \times 100 \\
& \text { Specificity }=\frac{T N}{T N+F P} \times 100 \\
& F-\text { Measure }=\frac{(2 * \text { Precision } * \text { Recall })}{(\text { Precision }+ \text { Recall })} \\
& \text { Accuracy }=\frac{T P+T N}{T P+T N+F P+F N} \times 100
\end{aligned}
$$

In next section, the enactment of proposed system is analyzed in relations of measureable and qualitative terms.

\section{i. Quantitative Analysis for Segmentation}

Table I. Expressions the proportional study of projected technique and two existing techniques by comparison of the both techniques with accuracy measurements and segmentation methods.

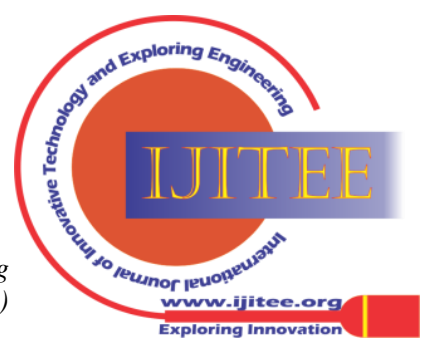


Pigeon Metheuristic Optimized Generative Adversarial Networks and ARKFCM Algorithms for retinal vessel Segmentation and Classification

Table - I Segmentation Performance Comparison Of The Remaining And Arkfcm Technique

\begin{tabular}{|l|l|l|}
\hline Author & Segmentation methodology & Accuracy (\%) \\
\hline $\begin{array}{l}\text { T. } \\
\text { Jebaseeli[24] }\end{array}$ & $\begin{array}{l}\text { K-means Clustering integrated } \\
\text { with FCM system }\end{array}$ & 96.83 \\
\hline $\begin{array}{l}\text { N.Eladawi, } \\
{[28]}\end{array}$ & Morphological Pyramid and FCM & 97.05 and 95.85 \\
\hline Proposed & ARKFCM & 97.18 \\
\hline
\end{tabular}

\section{B. Quantitative Analysis for Classification Algorithm}

The parameters achieved sensitivity, specificity f-measure, accuracy and Area under the curve (AUC), equated to the anticipated ordering mode. The performance of proposed and existing classification systems for the DRIVE and STARE dataset simulation is described in table 2 and 3.

Table - II Performing Analysis Of Proposed And Existing Classifiers By The Means Of Sensitivity, F-Measure, Accuracy And Specificity For Drive Dataset.

\begin{tabular}{|c|l|l|l|l|}
\hline Classifiers & $\begin{array}{l}\text { Sensitivity } \\
(\mathbf{\% )}\end{array}$ & $\begin{array}{l}\text { Specificity } \\
\mathbf{( \% )}\end{array}$ & $\begin{array}{l}\text { F-measur } \\
\mathbf{e}(\%)\end{array}$ & $\begin{array}{l}\text { Accurac } \\
\mathbf{y}(\%)\end{array}$ \\
\hline CNN & 73.90 & 84.23 & 79.78 & 80.25 \\
\hline $\begin{array}{c}\text { PIO optimized } \\
\text { CNN }\end{array}$ & 78.53 & 80.98 & 86.05 & 88.35 \\
\hline GAN & 77.01 & 85.54 & 83.32 & 86.47 \\
\hline $\begin{array}{c}\text { PIO optimized } \\
\text { Proposed } \\
\text { GAN }\end{array}$ & 86.41 & 90 & 89.84 & 92.77 \\
\hline
\end{tabular}

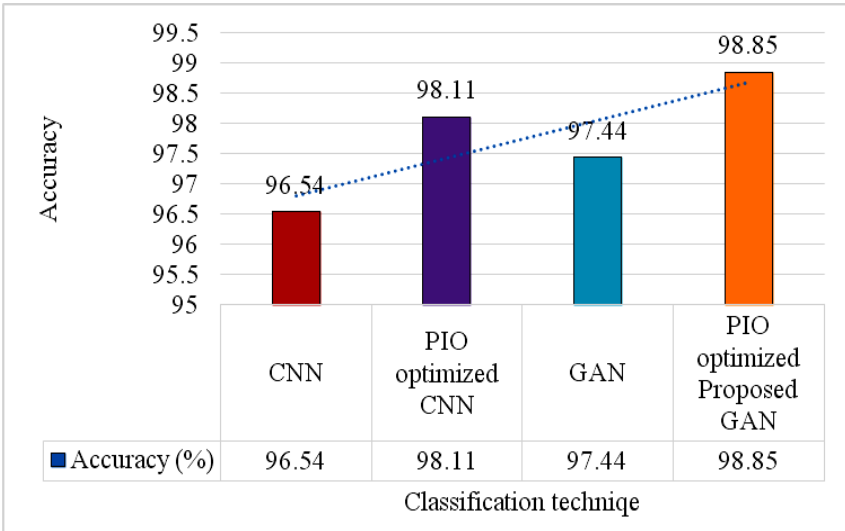

Fig. 8: Graphical comparison of proposed and existing classifiers by means accuracy with DRIVE dataset.

In above table 2 and figure 8 described that the comparisons of different classifier performance. There are totally four kinds of parameter metric are calculated such as sensitivity, f-measure, Accuracy and specificity. The evaluation is determined by using the DRIVE dataset. In first CNN classifier used to classify the outcomes as Sensitivity of $73.90 \%$, specificity of $84.23 \%$ and accuracy of $80.25 \%$. After we considered to use the PIO optimized CNN method to attain the accuracy of $88.35 \%$. It is slightly better than the CNN method. Then GAN classifier is used to evaluate the classification performance of F-measure value of 83.32 and accuracy of $86.47 \%$. It is better accuracy than CNN classifier. After we moved to enhance the accuracy by using proposed model of PIO optimized GAN scheme. This achieve the sensitivity of $86.41 \%$, specificity of $90 \%$, F-measure value of $89.84 \%$ and accuracy of $92.77 \%$. It is the better outcomes than the previous existing classification techniques.
Table - III. Enactment Enquiry That Proposed And Existing Classifiers By The Means Of Sensitivity, F-Measure, Accuracy And Specificity For Stare Dataset.

\begin{tabular}{|c|c|c|c|c|}
\hline $\begin{array}{c}\text { Classifier } \\
\text { s }\end{array}$ & $\begin{array}{c}\text { Sensitivity } \\
\text { (\%) }\end{array}$ & $\begin{array}{c}\text { Specificity } \\
\text { (\%) }\end{array}$ & $\begin{array}{c}\text { F-measure } \\
\text { (\%) }\end{array}$ & $\begin{array}{c}\text { Accuracy } \\
\text { (\%) }\end{array}$ \\
\hline CNN & 75.56 & 95.97 & 76.45 & 96.54 \\
\hline $\begin{array}{c}\text { PIO } \\
\text { optimized } \\
\text { CNN }\end{array}$ & 801.55 & 97.27 & 80.34 & 98.11 \\
\hline GAN & 81.16 & 97.55 & 79.86 & 97.44 \\
\hline $\begin{array}{c}\text { PIO } \\
\text { optimized } \\
\text { Proposed } \\
\text { GAN }\end{array}$ & 83.42 & 98.97 & 81.65 & 98.85 \\
\hline
\end{tabular}

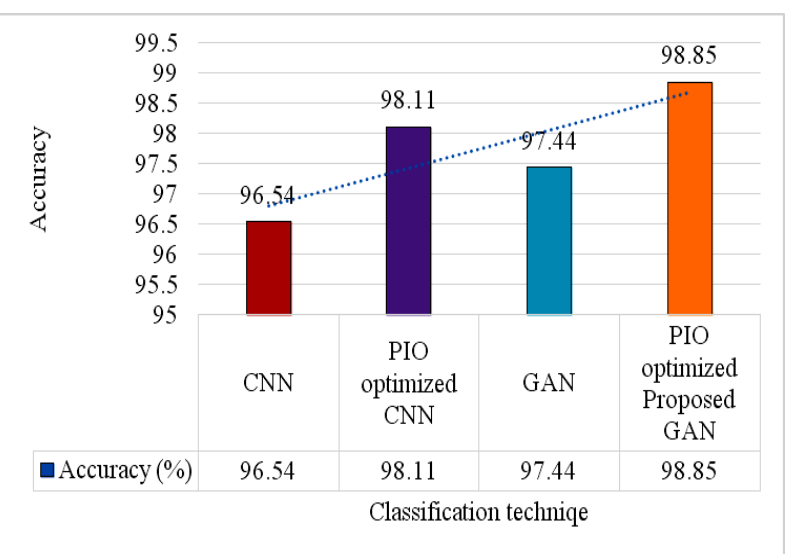

Fig. 9: Graphical comparison of proposed and existing classifiers by means accuracy with STARE dataset.

In above table 3 and figure 9 described that the comparisons of existing classifier with proposed scheme performance. The parameter metric are calculated by using the STARE dataset. In first CNN classifier used to classify the outcomes as Sensitivity of $75.56 \%$, specificity of $95.97 \%$ and accuracy of $96.54 \%$. After we considered to use the PIO optimized CNN method to attain the accuracy of $98.11 \%$. It is slightly better than the CNN method. Then GAN classifier is used to evaluate the classification performance of F-measure value of $79.86 \%$ and accuracy of $97.44 \%$. It is better accuracy than CNN classifier. After we moved to enhance the accuracy by using proposed model of PIO optimized GAN scheme. This achieve the sensitivity of $83.42 \%$, specificity of $98.97 \%$, F-measure value of $81.65 \%$ and accuracy of $98.85 \%$. It is the better performance than the previous existing classification techniques.

\section{CONCLUSION}

Generative Adversarial Networks (GAN) algorithm is introduced. Additionally to improve the proposed classification performance input image is transformed with the help of DWT. The DWT applied LL image and segmented images are cascaded. The cascade images are used for training and testing. The proposed system has validated with the help of DRIVE and STARE publically available datasets. The proposed system reaches the better results compared to existing systems.

Published By:

Blue Eyes Intelligence Engineering and Sciences Publication (BEIESP)

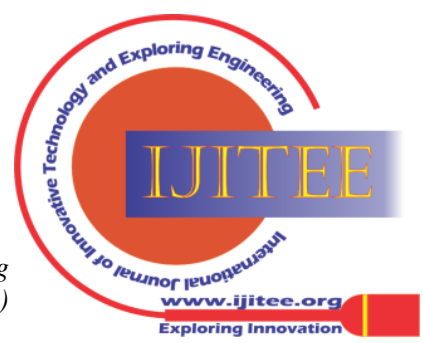


The papillae and blood vessels are separated to avoid blurring problems, so ophthalmologists examine the exudates. Diabetes causes many retinal diseases. It is impossible to classify as mild, moderate, and severe diseases in DR. In the future, the proposed system could be expanded to detect various eye diseases early in diabetic retinopathy. It was concluded that this work can be done by an ophthalmologist to analyze exudates of diabetic retinopathy, and this will help keep the patient from seeing loss.

\section{REFERENCES}

1. Wilkinson CP, Ferris III FL, Klein RE, Lee PP, Agardh CD, Davis M, Dills D, Kampik A, Pararajasegaram R, Verdaguer JT, Group GD, "Proposed international clinical diabetic retinopathy and diabetic macular edema disease severity scales," Ophthalmology, vol. 9, pp. 1677-82, sep 2003.

2. Fraz MM, Remagnino P, Hoppe A, Uyyanonvara B, Rudnicka AR, Owen CG, Barman SA,"Blood vessel segmentation methodologies in retinal images-a survey," Computer methods and programs in biomedicine. Vol. 108, no. 1, pp. 407-33, oct 2012

3. J. Nayak, P. S. Bhat, and U. R. Acharya, "Automatic identification of diabetic maculopathy stage using fundus images," J. Med. Eng.

4. K. Ram, G. D, Joshi, and J. Sivaswamy, "A successive clutterrejection-based approach for early detection of diabetic retinopathy," IEEE Trans. Biomed. Eng., vol. 58, no. 3, pp. 664-673, Mar. 2011.

5. Fraz MM, Remagnino P, Hoppe A, Uyyanonvara B, Rudnicka AR, Owen CG, Barman SA. An ensemble classification-based approach applied to retinal blood vessel segmentation. IEEE Transactions on Biomedical Engineering, vol. 59 no. 9, pp. 2538-48, jun 2012.

6. J. Nayak, P. S. Bhat, U. R. Acharya, C. M. Lim, and M. Kagathi, "Automated identification of diabetic retinopathy stages using digital fundus images," J. Med. Syst., vol. 32, pp. 107-115, 2008.

7. Osareh, B. Shadgar, and R. Markham, "A computationalintelligence-based approach for detection of exudates in diabetic retinopathy images," IEEE Trans. Inf. Technol. Biomed., vol. 13, no. 4, pp. 535-545, Jul. 2009.

8. M. U. Akram and S. A. Khan, "Multilayered thresholding-based blood vessel segmentation for screening of diabetic retinopathy," Eng. Comput., vol. 29, pp. 165-173, 2013.

9. P. Bankhead, C. N. Scholfield, J. G. McGeown, and T. M. Curtis, "Fast retinal vessel setection and measurement using wavelets and edge location refinement," PLOS One, vol. 7, no. 3, pp. 1-12, Mar. 2012.

10. J. Staal, M. D. Abramoff, M. Niemeijer, M. A. Viergever, and B. v. Ginneken, "Ridge-based vessel segmentation in color images of the retina," IEEE Trans. Med. Imag., vol. 23, no. 4, pp. 501-509, Apr. 2004.

11. M. S. Miri and A. Mahloojifar, "Retinal image analysis using curvelet transform and multistructure elements morphology by reconstruction," IEEE Trans. Biomed. Eng., vol. 58, no. 5, pp. 1183-1192, May 2011. from diabetic retinopathy images using fuzzy logic," IET Image Process., vol. 7, no. 2, pp. 121-130, 2013.

13. U. T. V. Nguyen, A. Bhuiyan, L. A. F. Park, and K. Ramamohanarao, "An effective retinal blood vessel segmentation method using multiscale line detection," Pattern Recogn., vol. 46, pp. 703-715, 2013.

14. S. Roychowdhury, D. D. Koozekanani, and K. K. Parhi, "DREAM: Diabetic retinopathy analysis using machine learning," IEEE J. Biomed. Health Inform., vol. 18, no. 5, pp. 1717-1728, Sep. 2014.

15. Y. Zhao, L. Rada, K. Chen, S. P. Harding, and Y. Zheng, "Automated vessel segmentation using infinite perimeter active contour model with hybrid region information with application to retinal images," IEEE Trans. Med. Imag., vol. 34, no.9, pp. 1797-1807, Sep. 2015.

16. T. Mapayi, S. Viriri, and J-R. Tapamo, "Comparative study of retinal Comput. Math. Method. M., vol. 2015, pp. 1-15, Nov. 2014.

17. E. Imani, M. Javidi, and H-R. Pourreza, "Improvement of retinal blood vessel detection using morphological component analysis," Comput. Meth. Prog. Bio., vol. 118, pp. 263-279, 2015.

18. A-S. Masoud, H. R. Pourreza, and T. Banaee, "A novel curvature-based algorithm for automatic grading of retinal blood Technol., vol. 33, no. 2, pp. 119-129, Feb. 2009.

12. N. G. Ranamuka and R. G. N. Meegama, "Detection of hard exudates vessel segmentation based on global thresholding techniques,"

vessel tortuosity," IEEE J. Biomed. Health Inform., vol. 20, no. 2, pp. 586-595, Mar. 2016.

19. S. Roychowdhury, D. D. Koozekanani, S. N. Kuchinka, and K. K. Parhi, "Optic disc boundary and vessel origin segmentation of fundus images," IEEE J. Biomed. Health Inform., vol. 20, no. 6, pp. 1562 1574, Nov. 2016.

20. P. Liskowski and K. Krawiec, "Segmenting retinal blood vessels with deep neural networks," IEEE Trans. Med. Imag., vol. 35, no. 11, pp. 2369-2380, Nov. 2016.

21. L. Seoud, T. Hurtut, J. Chelbi, F. Cheriet, and, J. M. P. Langlois, "Red lesion detection using dynamic shape features for diabetic retinopathy screening," IEEE Trans. Med. Imag., vol. 35, no. 4, pp. 1116-1126, Apr. 2016.

22. R. Pires, S. Avila, H. F. Jelinek, J. Wainer, E. Valle, and A. Rocha, "Beyond lesion-based diabetic retinopathy: a direct approach for retinal," IEEE J. Biomed, Health Inform., vol. 21, no. 1, pp. 193-200, Jan. 2017.

23. Verbraak, F.D.; Abramoff, M.D.; Bausch, G.C.F.; Klaver, C.; Nijpels, G.; Schlingemann, R.O.; van der Heijden, A.A. Diagnostic accuracy of a device for the automated detection of diabetic retinopathy in a primary care setting. Diabetes Care,vol. 42, pp. 651-656, 2019.

24. T. Jebaseeli, Jemima, C. Anand Deva Durai, and J. Dinesh Peter. "Segmentation of retinal blood vessels from ophthalmologic Diabetic Retinopathy images." Computers \& Electrical Engineering vol. 73 , pp. 245-258, 2019.

25. Pires, R.; Avila, S.; Wainer, J.; Valle, E.; Abramoff, M.D.; Rocha, A. A data-driven approach to referable diabetic retinopathy detection. Artif. Intell. Med, vol. 96, pp.93-106, 2019.

26. S. Dutta, Manideep, B. C., Basha, S. M., Caytiles, R. D., \&Iyengar, N. C. S. N. Classification of diabetic retinopathy images by using deep learning models. International Journal of Grid and Distributed Computing, vol. 11, no.1, pp. 89-106, 2019.

27. T. Jebaseeli, Jemima, C. Anand Deva Durai, and J. Dinesh Peter. "Segmentation of retinal blood vessels from ophthalmologic Diabetic Retinopathy images." Computers \& Electrical Engineering, vol. 73, pp. 245-258, 2019.

28. N. Eladawi, Elmogy, M., Khalifa, F., Ghazal, M., Ghazi, N., Aboelfetouh, A., ...\& ElBaz, A,"Early diabetic retinopathy diagnosis based on local retinal blood vessel analysis in optical coherence tomography angiography (OCTA) images," Medical physics, vol. 45, no. 10, pp. 4582-4599, 2018.

\section{AUTHORS PROFILE}

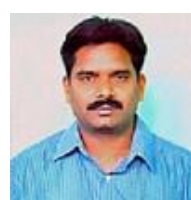

Dr. R. Kiran Kumar, received the M.Tech. Degree, from JNTUK, Kakinada. He received the Ph. D., (Computer Science and Engineering) from Acharya Nagarjuna University, India, March, 2009. He is working as Assistant Professor, in Department of Computer Science, University College, Krishna University. He is having more than 21 years of experience in teaching. His research interest includes AI, ML, DL, Image Processing and Computer vision.

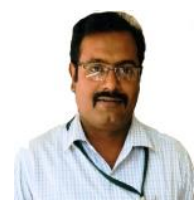

K. Arunabhaskar, Research Scholar, Department of Computer Science, Krishna University, Machilipatnam, received his M.Tech. Degree, from JNTUK, Kakinada in the year 2010. Working as Associate Professor, Aditya Engineering College, Aditya Nagar, Surampalem, East Godavari District, Andhra Pradesh, India. His research interest includes AI, ML, DL, Image Processing and Computer vision.

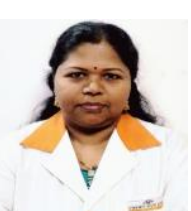

Dr. Ch Mani Mala, MBBS, DO, FCO, Chief Consultant - Phaco \& ICL Surgeon. She completed her MBBS from Andhra Medical College, Visakhapatnam in 1996, and DO from Rangaraya Medical College, Kakinada in 2004, Fellowship from Sri Kiran Eye Institute, Kakinada in 2005 and L.V.Prasad Eye Institute, Hyderabad in 2006. She is working as Chief Consultant - Phaco \& ICL Surgeon at Vasan Eye Care, Kakinada, East Godavari, Andhra Pradesh, India.

Published By: 